\title{
Simulations of the Beam-Beam Interaction with
}

\section{Transient or Stationary Beam Displacement}

D. Neuffer, A. Riddiford, A.G. Ruggiero

Fermilab, March 1982

\begin{abstract}
Results of beam-beam interaction simulations with a constant or transient or periodic displacement of the beam centers are described. No "beam blow-up" or significant emittance increase was observed. We conclude that the beam-beam interaction does not make colliding beams intrinsically unstable in constant or transient displacements at Tevatron parameters ${ }^{1}$.
\end{abstract}

\section{Introduction}

During colliding beam operation, significant displacements of colliding beam centers will occur either unintentionally due to collision misalignment errors or intentionally with deliberate Duam separation. In particular, it is conceivable to separate the 
beams in acceleration, and gradually to reduce their separation to centered collisions at full energy. It is important to determine whether beam instability occurs with constant or transient beam displacement.

In previous simulations of beam-beam interactions in the Tevatron 2,3 , the centers of the colliding beams were chosen to coincide. Simulations of two types of beam separation are reported here. First, a constant separation of beams was chosen, with separations of $0.1,0.3,0.5,1 .$, and 2 . times $\sigma$, the rms beam size. The lower values correspond to possible misalignment errors, the larger values to possible deliberate separations at unwanted crossings. Second, a transient separation was chosen, in which initially separated beams are combined over a finite number of turns $(1,000$ to 50,000$)$ by reducing their separation to zero. This simulates the reduction of separation at full energy described above.

\section{Simulation Procedure}

In our simulations we approximate particle circulation around the ring as the product of two transformations: a linear transport around the storage ring followed by a nonlinear beam-beam "kick" at the interaction area.

Transport around the ring can be represented by $2 \times 2$ matrices for both transverse ( $x$ and $y$ ) dimensions: 


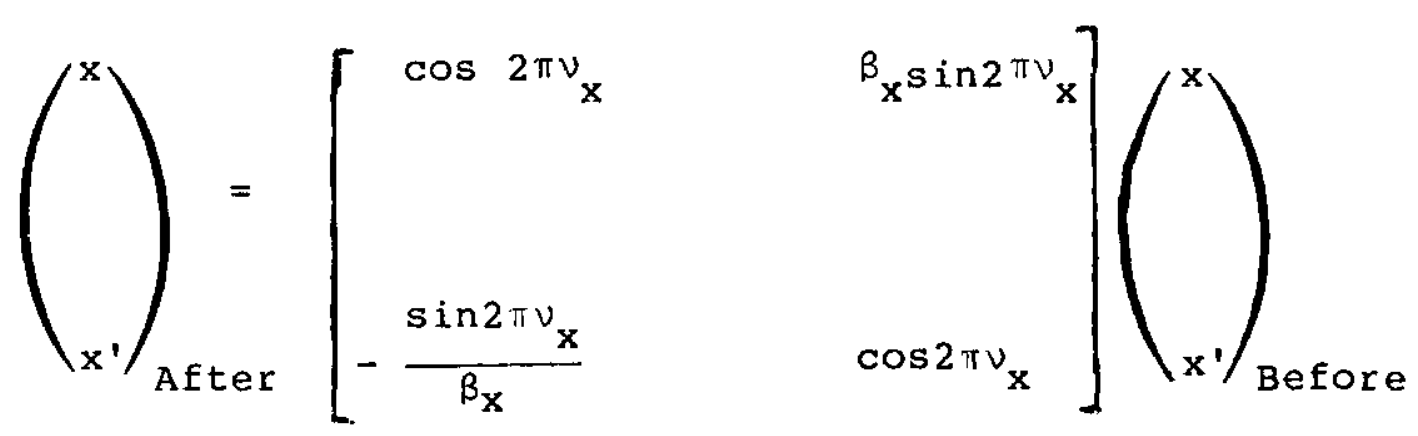

In this linear transport $x$ and $y$ motion are decoupled. $\nu_{x}$, $\nu_{y}$, $\beta_{x}, \beta_{y}$ are the usual courant-snyder tunes and beta-functions ${ }^{4}$. The beam-beam kick can be represented as

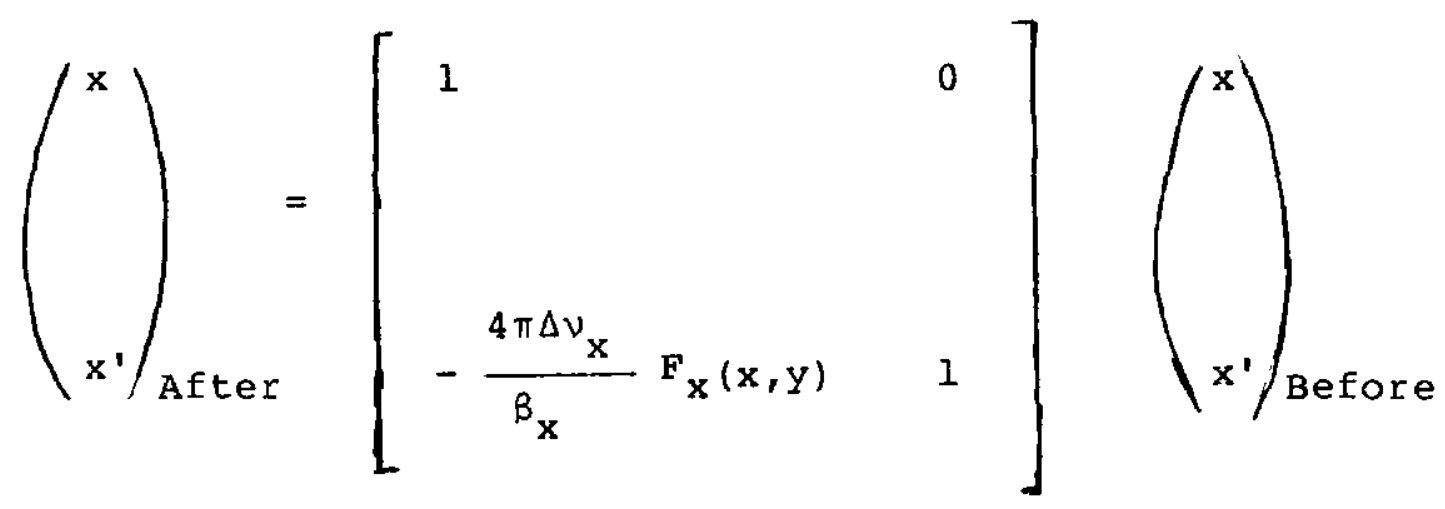

with a similar expression for $y, y^{\prime}$.

The product of these transformations is equivalent to integration of the equation of motion:

$$
x^{\prime}{ }^{\prime}+K_{x}(s) x=-\frac{4 \pi \Delta \nu_{x}}{\beta_{x}} F_{x}(x, y) x \delta_{p}(s)
$$

$s$, the distance along the storage $r i n g$, is the independent variable, $\delta_{p}(s)$ is a periodic delta-function, and $k_{x, y}(s)$ is the focusing function. 
In the present report we choose parameters which approximate the conditions ${ }^{1}$ in the Tevatron: $\beta_{x}=\beta_{y}=2 \mathrm{~m}$ and we choose

$$
F_{x}=F_{y}=\frac{1-e^{-\left(x^{2}+y^{2}\right) / 2 \sigma^{2}}}{\left(x^{2}+y^{2}\right) / 2 \sigma^{2}}
$$

with $\sigma=0.0816 \mathrm{~mm}$, which is the nonlinear force due to a round, gaussian charge distribution of rms radius $\sigma$.

Beam displacement is simulated by replacing $x$ by $x+x_{0}$ in the beam-beam force, where $x_{0}$ is the displacement of the beam centers. $\mathrm{F}_{\mathrm{X}}=\mathrm{F}_{\mathrm{Y}}$ is replaced by

$$
F=\frac{1-e^{-\left(\left(x+x_{0}\right)^{2}+y^{2}\right) / 2 \sigma^{2}}}{\left(\left(x+x_{0}\right)^{2}+y^{2}\right) / 2 \sigma^{2}}
$$

and $x \rightarrow x+x_{0}$ on the right hand side of equation (3) and $x+x+$ $x_{0}$ in equation (2) so that

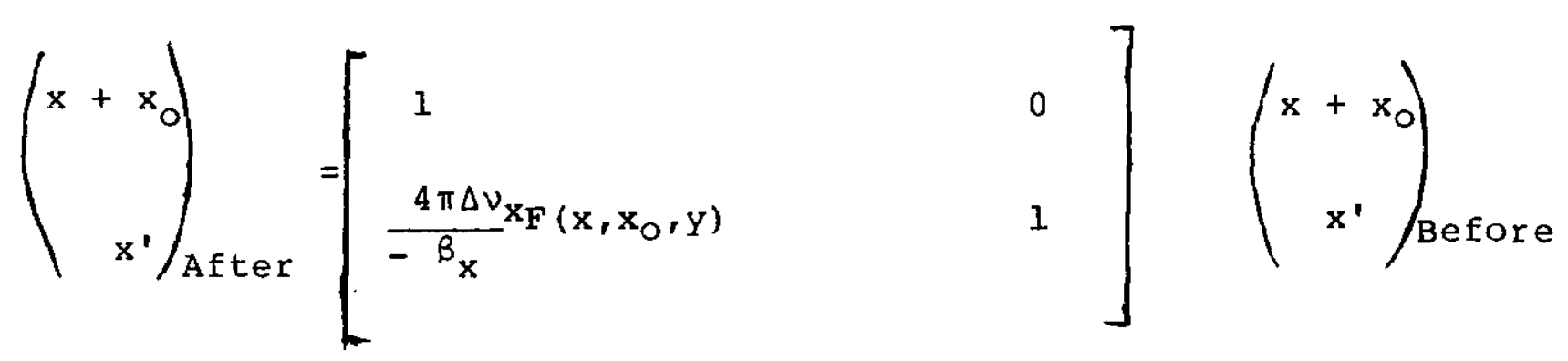


with a similar equation for $y_{,} y^{\prime}$ except $y_{O}=0$ is always chosen. $x_{0}$ is made a function of time to simulate transient displacement. In the simulations, 100 particle trajectories are calculated with initial positions $\left(x, x^{\prime}, y, y^{\prime}\right)$ chosen randomly, within a 4-D gaussian distribution determined by the rms size parameter $\sigma$ and, as described in reference 1, transported following the above matrices.

Every two thousand turns emittance values $\varepsilon_{x}, \varepsilon_{y}, \varepsilon_{R}$ are calculated using

$$
\begin{aligned}
& \varepsilon_{\mathrm{x}}=6 \sqrt{\left\langle(\mathrm{x}-\overline{\mathrm{x}})^{2}\right\rangle\left\langle(\mathrm{x}-\mathrm{x})^{2}\right\rangle} \\
& \varepsilon_{\mathrm{y}}=6 \sqrt{\left\langle(\mathrm{y}-\overline{\mathrm{y}})^{2}\right\rangle\left\langle\left(\mathrm{y}^{\prime}-\overline{\mathrm{y}}^{\prime}\right)^{2}\right\rangle} \\
& \varepsilon_{\mathrm{R}}=\sqrt{\varepsilon_{\mathrm{X}}^{2}+\varepsilon_{\mathrm{Y}}^{2}}
\end{aligned}
$$

and changes in these values as functions of time are followed.

"Doubling" times for $X, Y$, and $R$ emittances are obtained from the slopes of the best straight line fits for $\varepsilon_{x}, \varepsilon_{y}$, and $\varepsilon_{R}$ as functions of time from $t=0$, using rms emittance values calculated every 2000 turns, as described in reference 2 and 3 ; a slope b is obtained for each parameter. Also from the statistical fluctuations in emittances an error $\sigma_{b}$ is obtained. A slope $b$ is considered significantly different from zero if it is greater than $\sigma_{b}$ in magnitude. A "doubling time" $T_{x}$ is obtained by $T_{x}=\bar{x} / b_{x}$ and a "statistically significant doubling time" by $T_{\sigma_{x}}=\bar{x} / \sigma_{b_{x}}$. 
We present two sets of simulations. In the first set the beams are displaced a constant distance, and six million turns are calculated. In the second set, the transient case, the beams are displaced by 6 o for 200,000 turns, and the displacement then reduces to zero over 1-50 thousand turns. The centered beams are then tracked for another one million turns.

\section{Results of Constant Displacement simulations}

In tables 1-5, we display the statistical analysis of the 6 million turn simulations with displacements $x_{0}=.1 \sigma, .3 \sigma, .5 \sigma$, $\sigma$, and $2 \sigma$. The tunes $\nu_{x}, \nu_{y}$ were chosen at $\nu_{x}=.3439, \nu_{y}=0.1772$, in a "resonance-free" region and $\Delta v=.01, \Omega 3$ times larger than expected for $\mathrm{p}-\overline{\mathrm{p}}$ collisions.

As can be seen from the tables, the observed doubling times are greater than the "statistical error times" indicating that beam displacement does not lead to a linear change in $\varepsilon_{x}, \varepsilon_{y}$ or $\varepsilon_{r}$ in time; that is, no beam blow-up occurs.

There is one noticeable effect. With increasing $x_{0}$ in this set of simulations, the mean value of $x$-emittance increases slightly while the mean value of y-emittance decreases slightly. The mean value of $r$-emittance remains unchanged.

This effect is shown in Table 6 and displayed graphically in Figure 1. The effect is established within the first 200,000 turns; we note here that all simulations use the same 100 initial particle positions and therefore start with precisely the same 
emittances. The net effect is an apparent exchange between $x$ and $y$ emittances to make the beam "rounder" $\left(\varepsilon_{x}=\varepsilon_{y}\right)$ when the collisions become more off-center, at least up to 2 o. The net effect is up to 28 exchange at $x_{0}=2 \sigma$, of the same magnitude as the $x$ and $y$ emittance difference at the start of the simulation.

\section{Simulations with a Transient Displacement}

In Table 7, we display results of the simulations with a transient beam displacement. The results show no significant changes in any of the beam parameters, and show that there is no beam blow-up in the transition from "separated" beams (6o separation) to colliding beams, independent of the transition rate.

\section{Simulations with a Periodic Displacement}

Another possible type of beam displacement is a periodic displacement which could be due to sources such as power supply ripple or synchrotron oscillations with a non-zero dispersion crossing. We have undertaken two simulations to test this possibility. In these, beam displacement varied as 


$$
x_{o}(t)=x_{\max } \sin \frac{2 \pi n(t)}{N}
$$

where $x_{\max }$ is the maximum oscillation amplitude, $\mathrm{n}$ is the turn number and $\mathrm{N}$ is the oscillation period. We chose $\mathrm{N}=1000$ to approximate expected periods of synchrotron oscillations and power supply ripple in the Tevatron. We chose two values of $\mathrm{x}_{\max }$ :
a) $x_{\max }=0.02 \mathrm{~mm}$ which is $\wedge \sigma / 4$
b) $x_{\max }=0.1 \mathrm{~mm}$ which is $\sim 1.250$

The results of the simulations are displayed graphically in Figures 2 and 3. Each simulation included a total of 6 million turns, simulations of $\Omega 2$ Tevatron minutes.

We see no significant changes in $x, y$, or $r$ emittance, and extrapolate emittance doubling times of $>1$ day. This demonstrates that periodic crossing modulation with the beam-beam interaction does not lead to large beam blow-up at these parameters. 


\section{References}

1. The Fermilab Antiproton Source Design Report, February 1982 .

2. D. Neuffer, A. Riddiford, A. Ruggiero, FN-333, Fermilab, April 1981.

3. D. Neuffer, A. Riddiford, A. Ruggiero, FN-343, Fermilab, July 1981 .

4. E.D. Courant and H.S. Snyder, Annals of Physics, 3,1 (1958). 
TABLE 1 Enittance data for Case $C / X 0=0.1 \sigma, \sigma=0.08165 \mathrm{~mm}, \nu_{x}=0.3439, \nu_{y}=0.1772, \Delta \nu=0.01$

\begin{tabular}{|c|c|c|c|c|c|c|c|c|}
\hline $\begin{array}{l}\text { Million } \\
\text { fums }\end{array}$ & $\begin{array}{c}X \\
\text { Average } \\
(m m-m r a d)\end{array}$ & $\begin{array}{c}\mathrm{T}_{\mathrm{X}} \\
\text { Doubling } \\
\text { Time-X } \\
\text { (days) }\end{array}$ & $\begin{array}{c}Y \\
\text { Average } \\
(m-m r \gtrsim a)\end{array}$ & $\begin{array}{c}\mathrm{T}_{\mathrm{Y}} \\
\text { Doubling } \\
\text { Time-Y } \\
\text { (days) }\end{array}$ & $\begin{array}{c}\mathrm{R} \\
\text { Average } \\
(\mathrm{m} m-\mathrm{ma} a d)\end{array}$ & $\begin{array}{c}\mathrm{T}_{\mathrm{R}} \\
\text { Doubling } \\
\text { Tine-R } \\
\text { (days) }\end{array}$ & $\begin{array}{l}\text { Correlation } \\
\text { Bar Averaje }\end{array}$ & $\begin{array}{l}\text { Coefficient } \\
\text { Cumulative }\end{array}$ \\
\hline 0.2 & & $( \pm 0.0111)$ & & $( \pm 0.00933)$ & & $( \pm 0.0137)$ & & \\
\hline 0.2 & 0.0175712 & -0.0518 & 0.0187775 & -0.0150 & 0.0257176 & -0.0224 & 0.0640 & $0.06 \div 0$ \\
\hline 1.0 & 0.0175833 & 0.182 & 0.0187683 & -0.0794 & 0.0257191 & -0.242 & 0.0233 & -0.0124 \\
\hline 2.0 & 0.0175908 & 0.378 & 0.018 .605 & -0.233 & 0.0257185 & -0.969 & -0.1118 & 0.0082 \\
\hline 3.0 & 0.0175359 & -1.27 & 0.0187604 & -1.17 & 0.0257151 & -1.22 & 0.0013 & 0.0148 \\
\hline 4.0 & 0.0175825 & -0.802 & $0.018760 \mathrm{i}$ & -4.05 & 0.0257126 & -1.40 & 0.0116 & 0.0041 \\
\hline 5.0 & 0.0175844 & -9.66 & $0.018 ? 612$ & 6.84 & 0.0257147 & 34.8 & 0.1497 & 0.0275 \\
\hline 5.2 & 0.0175851 & 9.17 & 0.0187601 & -6.10 & 0.0257144 & -26.7 & 0.0466 & 0.0277 \\
\hline 5.4 & 0.0175852 & 9.17 & 0.0187604 & -13.1 & 0.0257145 & 37.8 & -0.0768 & 0.0233 \\
\hline 5.6 & 0.0175851 & 3.19 & 0.0187609 & 18.2 & 0.0257156 & 5.66 & -0.1233 & 0.0196 \\
\hline 5.8 & 0.0175372 & 1.78 & $0.018 ? 614$ & 5.92 & 0.0257168 & 2.84 & 0.1570 & 0.0248 \\
\hline 6.0 & 0.0175868 & $\begin{array}{c}2.42 \\
+1.70)\end{array}$ & 0.0187615 & 5.71 & 0.0257166 & 3.49 & -0.1904 & 0.0185 \\
\hline 6.0 & & & & $( \pm 1.69)$ & & $( \pm 2.43)$ & & \\
\hline
\end{tabular}


TABLE 2 Rmittance data for Case $c / x 0=0.3 \sigma, \sigma=0.08165 \mathrm{~mm}, \nu_{x}=0.3 \div 39, \nu_{y}=0.1772, \Delta \nu=0.01$

\begin{tabular}{|c|c|c|c|c|c|c|c|c|}
\hline $\begin{array}{l}\text { Willion } \\
\text { Turns }\end{array}$ & $\begin{array}{c}X \\
\text { Average } \\
(n m-m a d)\end{array}$ & $\begin{array}{c}\mathrm{T}_{\mathrm{X}} \\
\text { Doubling } \\
\text { Time-X } \\
\text { (d)ys) }\end{array}$ & $\begin{array}{c}Y \\
\text { Average } \\
\text { (nm-nrad) }\end{array}$ & $\begin{array}{c}\mathrm{T}_{\mathrm{Y}} \\
\text { Doubling } \\
\text { Time } \mathrm{Y} \\
\text { (dzys) }\end{array}$ & $\begin{array}{c}R \\
\text { Average } \\
(\text { min-inrad) }\end{array}$ & $\begin{array}{c}\mathrm{T}_{\mathrm{R}} \\
\text { Doubling } \\
\text { Time-i } \\
\text { (days) }\end{array}$ & $\begin{array}{l}\text { Correlation } \\
\text { Bar Average }\end{array}$ & $\begin{array}{l}\text { Coefficient } \\
\text { Curnulative }\end{array}$ \\
\hline 0.2 & & $( \pm 0.00928)$ & & $( \pm 0.00978)$ & & $( \pm 0.0135)$ & & \\
\hline 0.2 & 0.0176536 & -0.00811 & $0.018666 \varepsilon$ & -0.0113 & 0.0256932 & -0.00962 & 0.0128 & 0.0128 \\
\hline 1.0 & 0.0176617 & 0.250 & 0.0186773 & -0.199 & 0.0257069 & -1.40 & -0.0925 & -0.0626 \\
\hline 2.0 & 0.0176735 & 0.278 & 0.0186853 & 0.648 & 0.0257211 & 0.400 & 0.0057 & -0.0525 \\
\hline 3.0 & 0.0176714 & 18.2 & 0.0180925 & 0.401 & 0.0257245 & 0.750 & -0.0433 & -0.0618 \\
\hline 4.0 & 0.0176707 & $-17 \cdot 3$ & $0.018 \mathrm{rig}$ 't & 1.32 & 0.0257233 & 2.70 & -0.0302 & -0.0635 \\
\hline 5.0 & 0.0176648 & $-0,671$ & 0.0180881 & -2.66 & 0.0257163 & -1.11 & 0.0342 & -0.0346 \\
\hline 5.2 & 0.0176630 & -0.547 & $0.018688 ?$ & $-3 \cdot 31$ & 0.0257156 & -0.977 & 0.0344 & -0.0320 \\
\hline 5.4 & 0.0176636 & -0.686 & 0.0186871 & -1.85 & 0.0257152 & -1.03 & 0.0860 & -0.0281 \\
\hline 5.6 & 0.0176324 & -0.735 & 0.0186865 & -1.58 & 0.0257147 & -1.02 & -0.1940 & -0.0347 \\
\hline 5.8 & 0.017622 & -0.658 & 0.0180353 & $-1 \cdot 20$ & 0.0257130 & -0.864 & 0.0316 & -0.0313 \\
\hline 6.0 & $0.01 \cdots 60$ & -0.732 & 0.018683 & -0.922 & 0.0257122 & -0.852 & -0.1087 & -0.0342 \\
\hline 6,0 & & $( \pm 1,70)$ & & $( \pm 1.56)$ & & $( \pm 2.34)$ & & \\
\hline
\end{tabular}

\section{$:$}


TABIE 3 Emittance data for Case $C / X_{0}=0.5 \sigma, \sigma=0.08165 \mathrm{~mm}, \nu_{\mathrm{x}}=0.3439, \nu_{\mathrm{y}}=0.1772, \Delta \nu=0.01$

\begin{tabular}{|c|c|c|c|c|c|c|c|c|}
\hline $\begin{array}{l}\text { Million } \\
\text { Turns }\end{array}$ & $\begin{array}{c}X \\
\text { Averase } \\
\text { (min-inrad) }\end{array}$ & $\begin{array}{c}\mathrm{T}_{\mathrm{X}} \\
\text { Doubling } \\
\text { Time-X } \\
\text { (days) }\end{array}$ & $\begin{array}{c}Y \\
\text { Averzge } \\
(m n-m r a d)\end{array}$ & $\begin{array}{c}\mathrm{T}_{\mathrm{Y}} \\
\text { Doubling } \\
\text { Time- } \mathrm{Y} \\
\text { (days) }\end{array}$ & $\begin{array}{c}\mathrm{i} \\
\text { Average } \\
(\mathrm{mm}-\operatorname{mrad})\end{array}$ & $\begin{array}{l}\mathrm{T}_{\mathrm{R}} \\
\text { Doubling } \\
\text { Time-li } \\
\text { (dxys) }\end{array}$ & $\begin{array}{l}\text { Correlation } \\
\text { bar Average }\end{array}$ & $\begin{array}{l}\text { Coefficient } \\
\text { Cumulative }\end{array}$ \\
\hline 0,2 & & $( \pm 0.00983)$ & & $( \pm 0.00904)$ & & $( \pm 0.0135)$ & & \\
\hline 0.2 & 0.0177637 & -0.00734 & 0.0186398 & -0.123 & 0.0257499 & -0.00931 & -0.0141 & -0.0141 \\
\hline 1.0 & 0.0177518 & -0.0809 & 0.0186176 & -0.508 & 0.0257259 & -0.145 & -0.0833 & -0.0577 \\
\hline 2.0 & 0.0177595 & 0.737 & 0.0186242 & 1.13 & 0.0257365 & 0.891 & -0.1648 & -0.1014 \\
\hline 3.0 & 0.0177587 & -2.50 & $0.018 t 291$ & 0.535 & 0.0257389 & 1.26 & -0.1101 & -0.1080 \\
\hline 4.0 & 0.0177583 & -4.86 & $0.0186 i 26 !$ & -125 & 0.0257364 & -9.88 & -0.0664 & -0.0894 \\
\hline 5.0 & 0.0177581 & $-57 \cdot 5$ & 0.0186241 & -1.89 & 0.0257349 & -3.50 & -0.1738 & -0.0921 \\
\hline 5.2 & 0.0177591 & 4.33 & 0.018623 .5 & -1.59 & 0.0257351 & 4.54 & -0.0688 & -0.0914 \\
\hline 5.4 & 0.0177600 & 2.28 & 0.0186232 & -1.56 & 0.0257355 & -7.81 & -0.1069 & -0.0920 \\
\hline 5.6 & 0,0177602 & 2.22 & 0.0186 .3 .39 & -2.52 & 0.0257362 & 269 & -0.1637 & -0.0942 \\
\hline 5.8 & $0.01: 595$ & 4.75 & 0.0186255 & 39.1 & 0.0257369 & 9.04 & -0.0531 & -0.0936 \\
\hline 6.0 & 0.0177595 & 4.78 & 0.018026 & 8.15 & 0.0257373 & 6.22 & -0.2348 & -0.0976 \\
\hline 6.0 & & $( \pm 1.56)$ & & $( \pm 1.50)$ & & $( \pm 2.27)$ & & \\
\hline
\end{tabular}

命 
TALLE 4 Emittance data for Case $c / x_{0}=\sigma, \sigma=0.08165 \mathrm{~mm}, \nu_{x}=0.3439, \quad \nu_{y}=0.1772, \Delta \nu=0.01$

\begin{tabular}{|c|c|c|c|c|c|c|c|c|}
\hline $\begin{array}{l}\text { Million } \\
\text { Turns }\end{array}$ & $\begin{array}{c}x \\
\text { Average } \\
(m m-m x a d)\end{array}$ & $\begin{array}{c}\mathrm{T}_{\mathrm{X}} \\
\text { Doubling } \\
\text { Time-X } \\
\text { (days) }\end{array}$ & $\begin{array}{c}Y \\
\text { Average } \\
\text { (nam-lirad) }\end{array}$ & $\begin{array}{c}\mathrm{T}_{\mathrm{Y}} \\
\text { Doubling } \\
\text { Time-Y } \\
\text { (dzys) }\end{array}$ & $\begin{array}{c}R \\
\text { Averzage } \\
(\text { inn-madi) }\end{array}$ & $\begin{array}{c}\mathrm{T}_{\mathrm{R}} \\
\text { Doubling } \\
\text { Time-R } \\
\text { (days) }\end{array}$ & $\begin{array}{l}\text { Correlation } \\
\text { Bar Average }\end{array}$ & $\begin{array}{l}\text { Coefficient } \\
\text { Cunulative }\end{array}$ \\
\hline 0.2 & & $( \pm 0.00781)$ & & $( \pm 0.00865)$ & & $( \pm 0.0123)$ & & \\
\hline 0.2 & 0.0170439 & -0.0139 & $0.018492 i$ & -0.0109 & 0.0257692 & -0.0123 & -0.106 & -0.106 \\
\hline 1.0 & 0.0179583 & 0.0858 & 0.0185040 & -0.582 & 0.0257878 & 0.205 & -0.250 & -0.201 \\
\hline 2.0 & 0.0179522 & -0.874 & 0.0185084 & 31.9 & $0.025736 ?$ & -1.89 & -0.342 & -0.200 \\
\hline 3.0 & 0.0179499 & $-0.7^{4} 44$ & $0.018 \% 096$ & 2.11 & 0.0257859 & $-2 \cdot 34$ & -0.186 & -0.193 \\
\hline 4.0 & 0.0179490 & -1.12 & 0.0185070 & $-1,42$ & 0.0257834 & $-1 \cdot 28$ & -0.246 & -0.201 \\
\hline 5.0 & 0.0179474 & -1.36 & $0.018509 i$ & 3.88 & 0.0257844 & -4.76 & -0.118 & -0.201 \\
\hline 5.2 & 0.0179473 & -1.45 & 0.0185084 & -9.04 & 0.0257834 & -2.66 & -0.276 & -0.204 \\
\hline 5.4 & 0.0179492 & -9.31 & 0.0185091 & 20.8 & 0.0257851 & -52.9 & -0.069 & -0.200 \\
\hline 5.6 & 0.019 .94 & -14.4 & 0.0135101 & 3.28 & 0.0257860 & 7.65 & -0.419 & -0.206 \\
\hline 5.8 & 0.01 .95 & -63.7 & 0.0185081 & -5.12 & 0.0257846 & -9.84 & -0.1832 & -0.206 \\
\hline 6.0 & 0.0173 .89 & -5.97 & $0.018: 023$ & -7.39 & 0.0257843 & -6.95 & -0.180 & -0.205 \\
\hline 6.0 & & $( \pm 1.30)$ & & $( \pm 1.32)$ & & $( \pm 2.09)$ & & \\
\hline
\end{tabular}


TABLE 5 Enittance data for Case $c / x_{0}=2 \sigma, \sigma=0.08165 \mathrm{~mm}, \nu_{x}=0.3439, \nu_{y}=0.1772, \Delta \nu=0.01$

\begin{tabular}{|c|c|c|c|c|c|c|c|c|}
\hline $\begin{array}{l}\text { Hillion } \\
\text { Turns }\end{array}$ & $\begin{array}{c}\mathrm{X} \\
\text { Average } \\
(\text { min-mrad })\end{array}$ & $\begin{array}{c}\mathrm{T}_{\mathrm{X}} \\
\text { Doubling } \\
\text { Time-X } \\
(\text { days })\end{array}$ & $\begin{array}{c}Y \\
\text { Average } \\
(\text { mm-mad })\end{array}$ & $\begin{array}{c}\mathrm{T}_{Y} \\
\text { Doubling } \\
\text { Time-Y } \\
\text { (days) }\end{array}$ & $\begin{array}{c}\text { In } \\
\text { Average } \\
(\text { mnnmad) }\end{array}$ & $\begin{array}{c}\mathrm{T}_{\mathrm{R}} \\
\text { Doubiing } \\
\text { Time-R } \\
\text { (days) }\end{array}$ & $\begin{array}{l}\text { Correlation } \\
\text { Bar Average }\end{array}$ & $\begin{array}{l}\text { Coefficient } \\
\text { Cunulative }\end{array}$ \\
\hline 0.2 & & $( \pm 0.00744)$ & & $( \pm 0.00744)$ & & $( \pm 0.0125)$ & & \\
\hline $0 . ?$ & 0.0179733 & -0.0104 & 0.0183794 & -0.0215 & 0.0257091 & -0.0141 & -0.286 & -0.236 \\
\hline 1.0 & 0.0180010 & 0.138 & 0.0183733 & 3.56 & 0.0257247 & 0.274 & -0.194 & -0.253 \\
\hline 2.0 & 0.0179969 & -1.17 & 0.0183802 & 0.802 & 0.0257263 & 4.45 & -0.252 & -0.250 \\
\hline 3.0 & 0.0179993 & 1.79 & $0.018: 8821$ & 1.59 & 0.0257293 & 1.70 & -0.336 & -0.272 \\
\hline 4.0 & 0.0179949 & -0.996 & $0.018388^{4}$ & 0.722 & 0.0257301 & 4.66 & -0.283 & -0.282 \\
\hline 5.0 & 0.0179011 & -0.777 & 0.0133903 & 0.719 & 0.0257294 & 14.9 & -0.216 & -0.279 \\
\hline 5.2 & 0.0179913 & -0.921 & 0.0183901 & 0.843 & 0.0257294 & 15.0 & -0.203 & -0.275 \\
\hline 5.4 & 0.0179009 & $-0,944$ & 0.0185918 & 0.668 & 0.0257304 & 4.22 & -0.215 & -0.273 \\
\hline 5.6 & $0.01,7009$ & -1.05 & $0.018: 907$ & 0.922 & 0.0257296 & 12.3 & -0.475 & -0.280 \\
\hline 5.8 & $0.01,9920$ & -1.67 & $0.018391 j$ & 0.856 & 0.0257309 & 3.35 & -0.376 & -0.283 \\
\hline 6.0 & $0.01 \% \mathrm{~m}$ ? & -1.65 & 0.0182918 & 0.392 & 0.0257309 & 3.68 & -0.230 & -0.282 \\
\hline 6.0 & & $( \pm 1.36)$ & & $\left( \pm^{+} .25\right)$ & & $( \pm 2.17)$ & & \\
\hline
\end{tabular}


Table 6

Six Million Turn Mean Emittances

\begin{tabular}{|c|c|c|c|}
\hline Displa & $\bar{\varepsilon}_{x}$ & $\bar{\varepsilon}_{Y}$ & $\bar{\varepsilon}_{R}$ \\
\hline $0.1 \sigma$ & .017587 & .018762 & .025717 \\
\hline $0.3 \sigma$ & .017663 & .018684 & .025712 \\
\hline $0.5 \sigma$ & .017760 & .018626 & .025737 \\
\hline $1.0 \sigma$ & .017949 & .018508 & .025784 \\
\hline $2.0 \sigma$ & .017992 & .018292 & .025731 \\
\hline
\end{tabular}


PABif 7 Enitance datz for transient xo. Tor 200,000 turas $x 0=6 \sigma ; \sigma=0.06105 \mathrm{mr}$. Then $x 0$ was brought linearly to zero in $1000 ; 10,000 ; 50,000$ turns. The enittances were followed for another million turns, The transient numbers below refer to the transient turns only. The sums were begun again at the end of the transient, and are then cumulative.

\begin{tabular}{|c|c|c|c|c|c|c|c|c|c|c|}
\hline $\begin{array}{l}\text { Thousand } \\
\text { Turns }\end{array}$ & $\underset{(\text { Average }}{x}$ & $\begin{array}{l}\text { Doubling } \\
\left(\begin{array}{c}\text { (dime } \\
\text { (a) }\end{array}\right.\end{array}$ & $\begin{array}{l}\text { Significant } \\
\text { (dine-x } \\
\text { (diys) }\end{array}$ & $\begin{array}{c}Y \\
\text { (Average) }\end{array}$ & $\begin{array}{l}\text { Doubling } \\
\text { (Tine-Y } \\
\text { (days) }\end{array}$ & $\begin{array}{l}\text { Significant } \\
\text { (Time-Y }\end{array}$ & $\begin{array}{c}R \\
\text { (Averageras) }\end{array}$ & $\begin{array}{l}\text { Doubling } \\
(\text { dingerin }\end{array}$ & \multicolumn{2}{|c|}{$\begin{array}{l}\text { Significant } \\
\text { (Time-R }\end{array}$} \\
\hline $1 T$ & 0.0178043 & $0.00017^{4}$ & 0.000111 & 0.0184525 & -0.000068 & 0.00048 & 0.0256426 & -0.000214 & \multicolumn{2}{|c|}{0.000117} \\
\hline 201 & 0.0175502 & 0.0120 & 0.107 & 0.0187033 & 0.0076 & 0.123 & 0.0256494 & .0 .0093 & \multicolumn{2}{|l|}{0.176} \\
\hline 401 & 0.0175461 & -0.235 & 0.108 & 0.0186673 & -0.0174 & 0.113 & 0.0256202 & -0.0304 & \multicolumn{2}{|l|}{0.155} \\
\hline 601 & 0.0175560 & 0.0449 & 0.114 & 0.0186683 & -0.0589 & 0.113 & 0.0256276 & -0.235 & \multicolumn{2}{|l|}{0.156} \\
\hline 801 & 0.0175603 & 0.0806 & 0.112 & 0.0186791 & 0.139 & 0.118 & 0.0256383 & 0.105 & \multicolumn{2}{|l|}{0.159} \\
\hline 1001 & 0.0175545 & -6.09 & 0.111 & 0.0186771 & 3.08 & 0.117 & 0.0256330 & 16.5 & \multicolumn{2}{|l|}{0.159} \\
\hline $10 T$ & 0.0176651 & -0.000527 & 0.000633 & 0.0185461 & 0.000081 & 0.000466 & 0.0256142 & 0.000180 & \multicolumn{2}{|l|}{0.000823} \\
\hline 210 & 0.0175219 & 0.00678 & 0.100 & 0.0186992 & 0.00559 & 0.110 & 0.0256269 & 0.00614 & \multicolumn{2}{|l|}{0.146} \\
\hline 410 & 0.0175556 & 0.0145 & 0.114 & 0.0186787 & 0.411 & 0.110 & 0.0256349 & 0.0301 & \multicolumn{2}{|l|}{0.159} \\
\hline 61.0 & 0.0175620 & 0.0305 & 0.115 & 0.0186801 & 0.343 & 0.111 & 0.0256403 & 0.0599 & \multicolumn{2}{|l|}{0.159} \\
\hline 810 & 0.0175659 & 0.0506 & 0.119 & 0.0186880 & 0.0791 & 0.111 & 0.0256487 & 0.0631 & \multicolumn{2}{|l|}{0.160} \\
\hline 1010 & 0.0175650 & 0.101 & 0.121 & 0.0186863 & 0.160 & 0,107 & 0.0256469 & 0.126 & \multicolumn{2}{|l|}{0.158} \\
\hline $50 \mathrm{~T}$ & 0.0176049 & 0.00158 & 0.00242 & 0.0186692 & 0.000713 & 0.00223 & 0.0256624 & 0.000959 & \multicolumn{2}{|l|}{0.00385} \\
\hline 250 & 0.0176341 & -0.0163 & 0.141 & 0.01856477 & 0.00530 & 0.0959 & 0.0256061 & 0.0144 & \multicolumn{2}{|l|}{0.165} \\
\hline 450 & 0.0176041 & -0.0158 & 0.131 & 0.0185888 & 0.01 .01 & 0.107 & 0.0256103 & 0.0461 & \multicolumn{2}{|l|}{0.173} \\
\hline 650 & 0.0176091 & -0.0800 & 0.138 & 0.0186201 & 0.0168 & 0.106 & 0.0256291 & 0.0394 & \multicolumn{2}{|l|}{0.174} \\
\hline 850 & 0.0176034 & -0.0738 & 0.125 & 0.0186254 & 0.0352 & 0.105 & 0.0256291 & 0.116 & \multicolumn{2}{|l|}{0.169} \\
\hline 1050 & 0.0176046 & -0.205 & 0.127 & 0.0186330 & 0.0436 & 0.109 & 0.0256353 & 0.102 & 0.174 & $E$ \\
\hline & & & & & & & & & & \\
\hline
\end{tabular}


Figure 1

Six million turn mean emittances 28 a function of beat displacement.

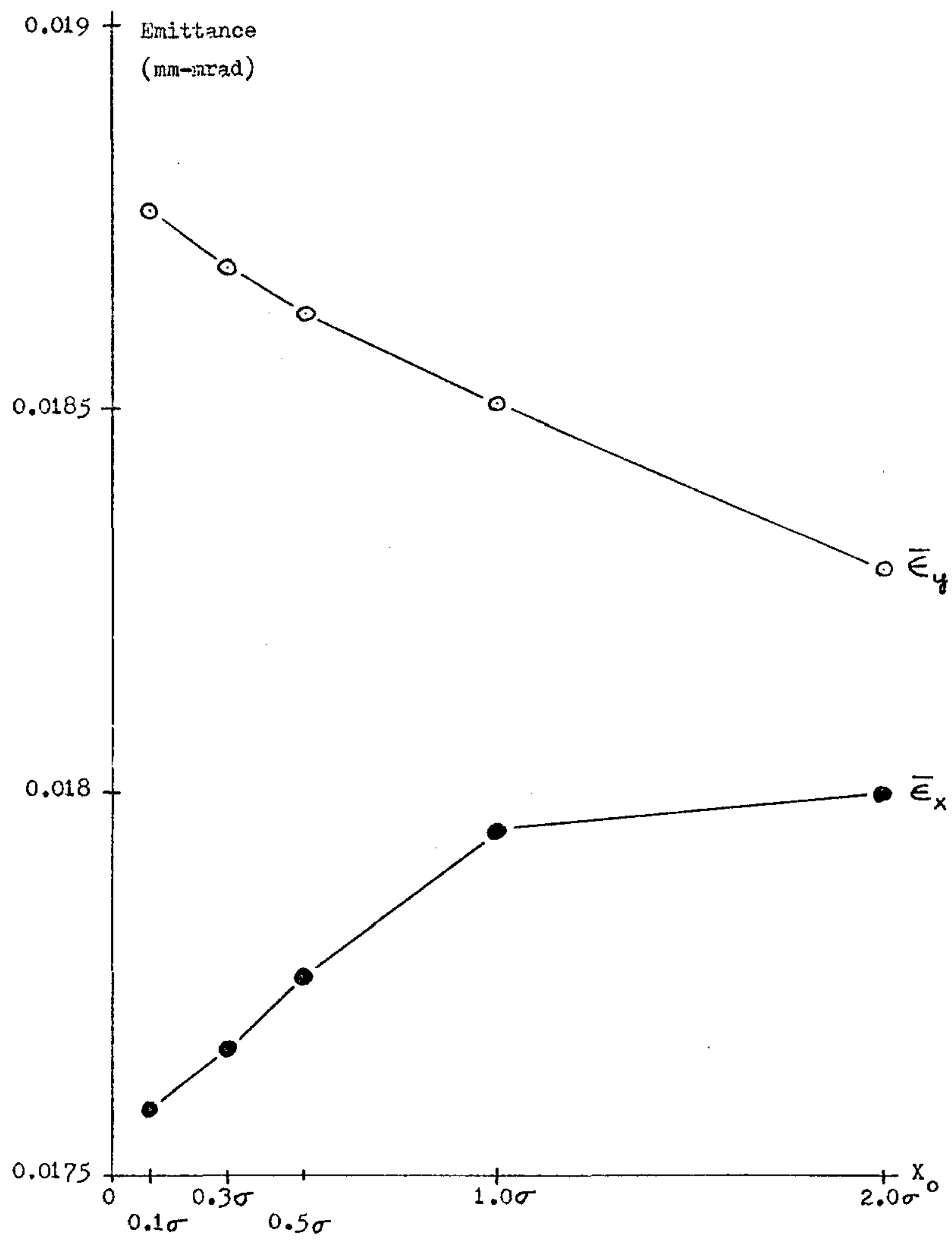


Figure 2
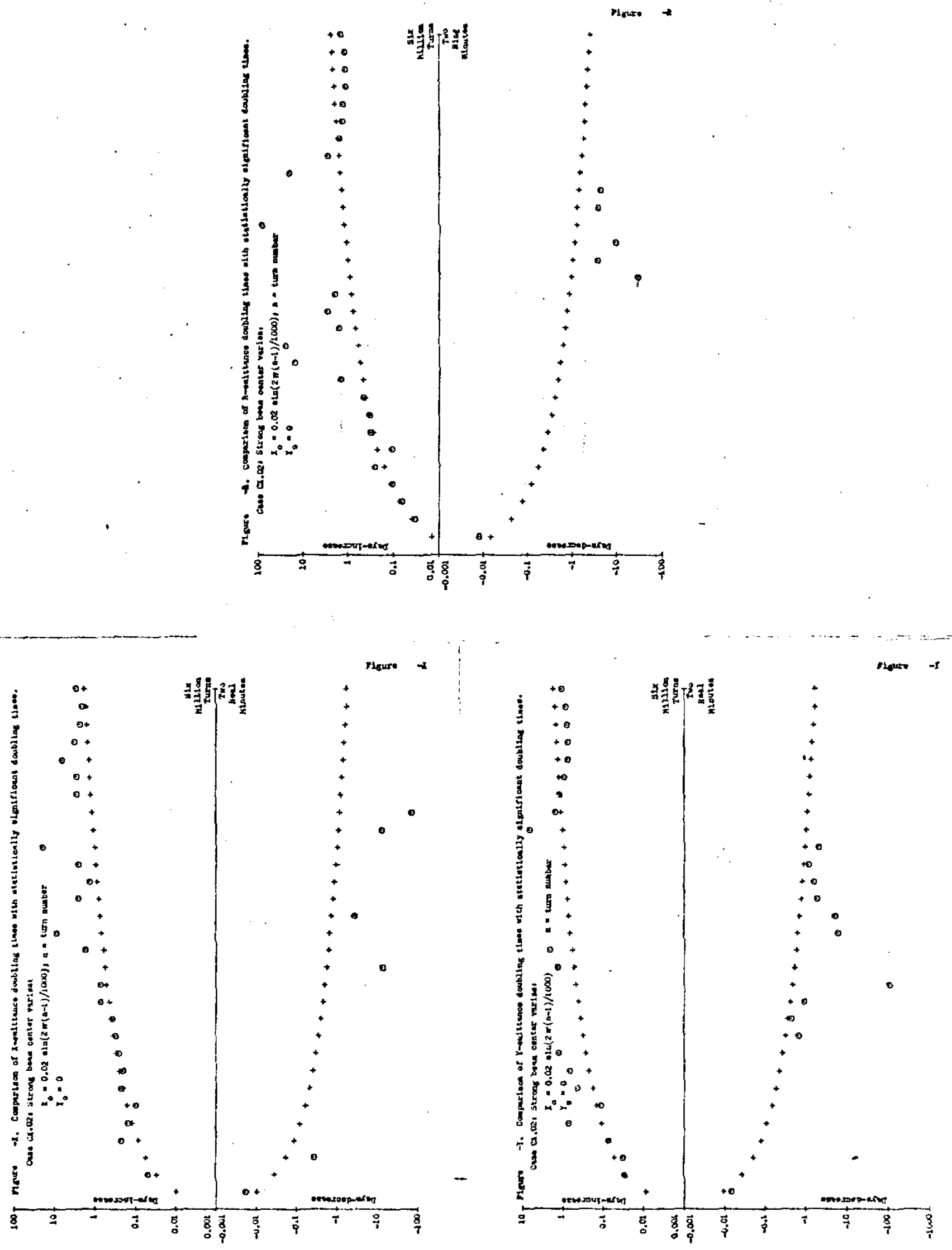


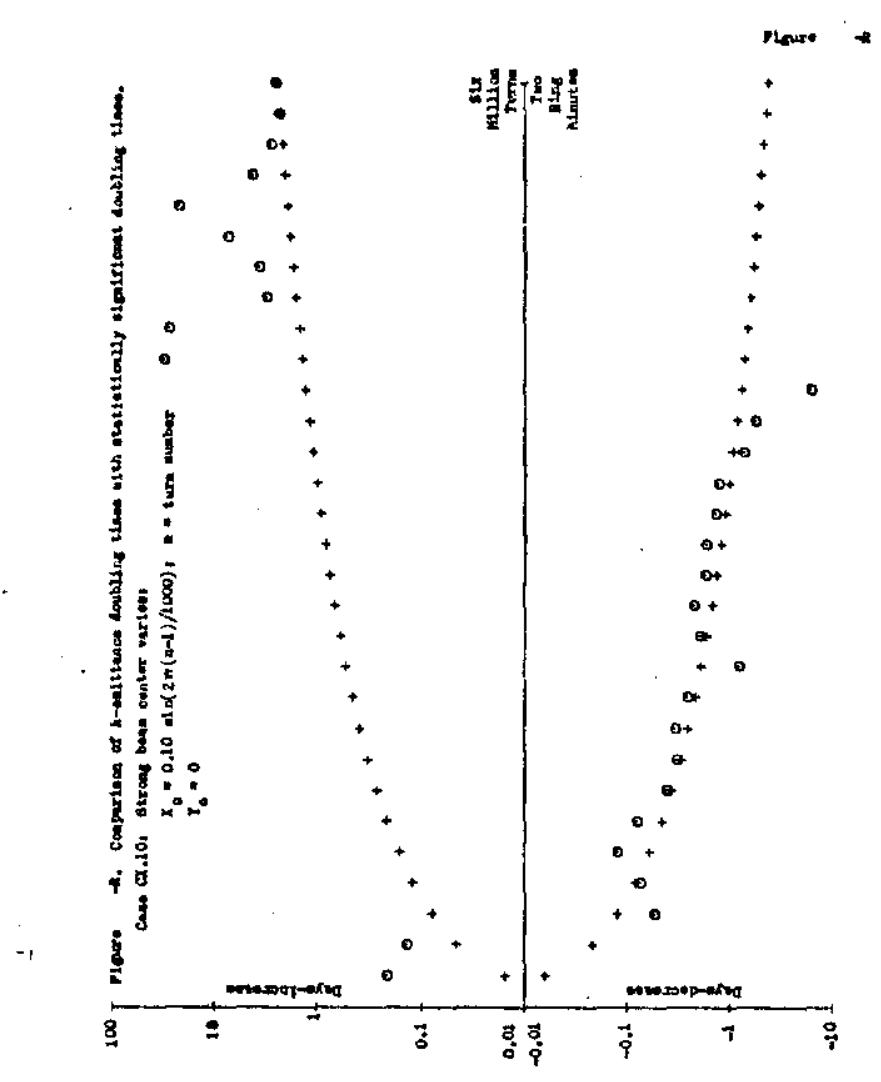

Figure 3

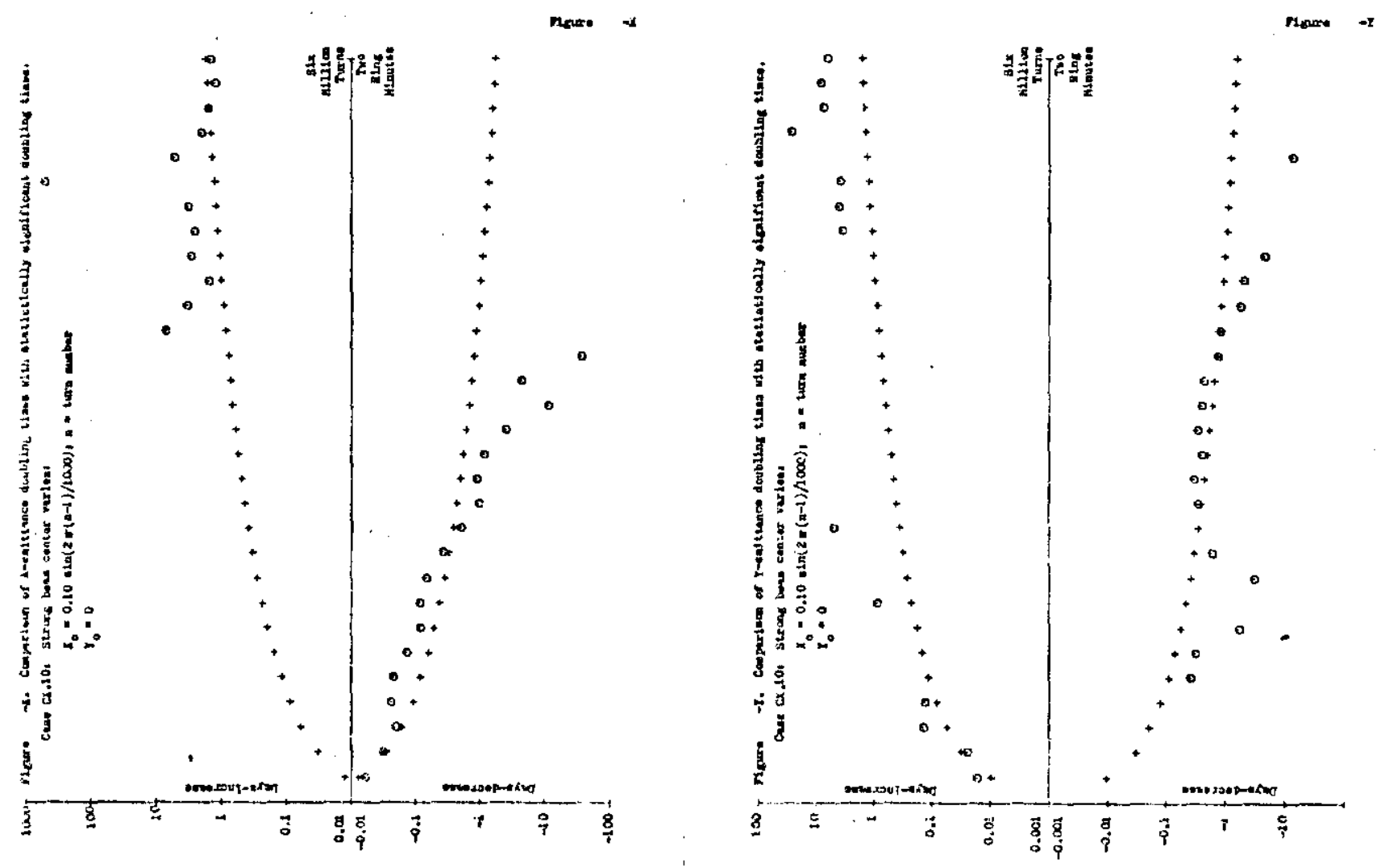

